\title{
Erratum
}

\section{Factual Versus Representational Utilities and their Interdimensional Comparisons}

Th. Bezembinder

Department of Psychology, Catholic University, Nijmegen, The Netherlands

P. van Acker

Department of Psychology, University of Geneva, Switzerland

Soc Choice Welfare (1987) 4:79-104

On p. 85 , the last line in the first paragraph in Sect. 4 should have been:

$$
1 \leq k \leq n \text { : }
$$

Please correct. 